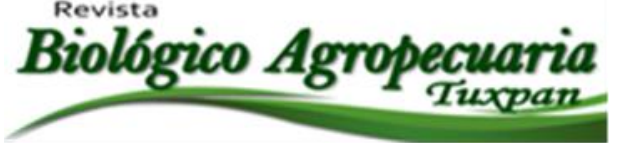

\title{
Uso de herramientas para mejorar la calidad de vida en el Municipio de Julimes a través de cooperativas agrícolas
}

Use of tools to improve the quality of life in the Municipality of Julimes through agricultural cooperatives

Jaime-Talamantes Mónica Arlette ${ }^{1}$, Leyva-Chávez Arwell Nathán ${ }^{\circledR}$, Ortega-Rodríguez Anabel², García-Muñoz Silvia Amanda², Piña-Ramírez Francisco Javier².

${ }^{1}$ Licenciatura en Desarrollo Territorial de la Facultad de Ciencias Agrotecnológicas, Universidad Autónoma de Chihuahua. ${ }^{2}$ Profesor de la Facultad de Ciencias Agrotecnológicas, Universidad Autónoma de Chihuahua Campus I, Chihuahua, Chih., México. Tel. (614)4391844, C.P. 31000.

${ }^{\circledR}$ Autor para correspondencia: nleyva@uach.mx

Recibido: $27 / 08 / 2020$

Aceptado: $19 / 10 / 2020$

\section{RESUMEN}

Uno de los principales problemas de las pequeñas unidades agrícolas rurales es establecer e implementar un modelo de gestión para aprovechar sus potencialidades, e impulsar el desarrollo productivo. El siguiente trabajo plantea la unión de los pequeños productores del Municipio de Julimes, Chihuahua, para la formación de una cooperativa agrícola, la cual pueda agregarles el valor correspondiente a los productos, conservándolos para poder comercializarlos y tenerlos como una herramienta sustentable de desarrollo dentro del municipio. Para determinar los factores que inciden en la producción se crearon mapas, así como los análisis de suelos para identificar los sistemas de cultivos y caracterizar las unidades agropecuarias, para el análisis de los diferentes factores, se utilizó un análisis descriptivo combinando herramientas de investigación. Se definió una muestra de 87 productores, a los cuales se les aplicó una encuesta debidamente formulada. Los resultados señalan, que el producto que se cosecha con mayor regularidad es el chile, mientras que éste es el que genera más ingresos, los productores opinaron que dentro de la población, existe una persona intermediaria al momento de vender sus productos, por lo cuál, optan por pertenecer y participar en una cooperativa agrícola.

Palabras Clave: Cooperativa agrícola, productores, desarrollo local, actividades económicas.

\begin{abstract}
One of the main problems of small rural agricultural units is to establish and implement a management model to take advantage of their potential and boost productive development. This thesis presents the
\end{abstract}


union of small producers of the municipality of Julimes, Chihuahua, for the formation of an agricultural cooperative that, as a result, can add value to the products and be able to commercialize them and have them as a sustainable development tool within the municipality. To determine the factors that affect production, maps were created, as well as soil analyzes to identify crop systems and characterize agricultural units. For the analysis of the different factors, a descriptive analysis was used, combining research tools. A sample of 87 producers was defined, to which a properly formulated survey was applied. The results indicate that the product that is harvested most regularly is chili, while this is the one that generates the most income; the producers believed that within the population, there is an intermediary person when selling their products, which is why, they choose to belong and participate in an agricultural cooperative.

Keywords: Agricultural cooperative, producers, local development, economic activities.

\section{INTRODUCCIÓN}

Con el paso del tiempo en América Latina se ha observado que las políticas económicas y sociales implementadas por los Estados y Municipios, se han centralizado en el desarrollo de lo urbano generando una degradación cada vez más profunda de lo rural. El desarrollo territorial se ha tomado como una alternativa eficaz para dinamizar lo rural, ofreciendo la oportunidad a los habitantes de participar en la búsqueda de soluciones para una mejor calidad de vida. Las cooperativas agropecuarias se consideran una vía exitosa para generar un desarrollo territorial, dado que la estructura incluyente de las cooperativas suma la formación de socios del municipio creando herramientas para ser activos en sus territorios. Éstas cooperativas son empresas sociales que en los territorios dinamizan la economía local y representan a la población rural ante el Estado y las empresas privadas (Favi \& Martínez, 2013).

El fin de la investigación es analizar y describir la producción y consumo de los recursos existentes, para diseñar una propuesta para la creación de una cooperativa agrícola y contribuir en una mejor calidad de vida de los habitantes del Municipio de Julimes, Chihuahua.

\section{MATERIALES Y MÉTODOS}

Para el análisis de los diferentes entornos, social, económico, y ambiental, del municipio de Julimes, Chihuahua, se realizó una investigación, de la cual se hicieron diferentes descargas de la página del Instituto Nacional de Estadística y Geografía (INEGI), y la Comisión Nacional para el Conocimiento y Uso de la Biodiversidad (CONABIO). Los datos utilizados fueron los de población, densidad de población, proyecciones de población, actividades económicas y turismo. $\mathrm{La}$ investigación incluyó actividades descriptivas, combinando herramientas de investigación, tales como: la recopilación de información secundaria de fuentes como artículos relacionados, revisas, sitios web, tesis, entre otros. Esta investigación se realizó utilizando un estudio descriptivo, puesto que se realiza en la realidad existente.

Población muestra. La población a estudiar son los productores del municipio de Julimes Chihuahua, que desarrollen o pretendan desarrollar algún cultivo en la región. Se debe 
tomar en cuenta que en el municipio existen 112 productores. Tamaño de muestra. Donde $N$ es el total de la población, $Z$ es el valor obtenido mediante niveles de confianza de la distribución
Normal, $e$ es el límite aceptable de error muestral y $\sigma$ es la desviación estándar.

Datos:

$$
\begin{array}{ccc}
N=112 & \begin{array}{c}
e=.05 \\
n=\frac{(112)(3.8416)(.25)}{(112-1)(.0025)+(.25)(3.8416)}
\end{array} &
\end{array}
$$

Figura 1. Fórmulas a utilizar.

Fuentes e instrumentos. Se elaboraron mapas con el sistema de información geográfica (ARCMAP), se obtuvo información del anuario estadístico geográfico 2017 y se descargaron archivos SHP de la Comisión Nacional para el Conocimiento y Uso de la Biodiversidad (CONABIO).

Técnicas de análisis. Se elaboró una encuesta, en la cuál se utilizaron dos variables, las cualitativas y las cuantitativas, con diferentes escalas. Se crearon ocho preguntas para la encuesta.

Se realizó el análisis de las encuestas y se condenso la información por medio de tablas y gráficas a través del programa Microsoft Office Excel 2013 para facilitar la comprensión de los datos obtenidos, identificando el número de productores y sus ingresos.

\section{RESULTADOS Y DISCUSIÓN}

La agricultura engloba a un $80 \%$ de los terrenos agrícolas en el Municipio de Julimes, esto equivale a 13,066 ha en promedio. El producto con más siembra por año es el chile con un $44 \%$, alfalfa $20 \%$, maíz $18 \%$, cebolla $10 \%$ y la sandía con un $5 \%$ (Figura 2).

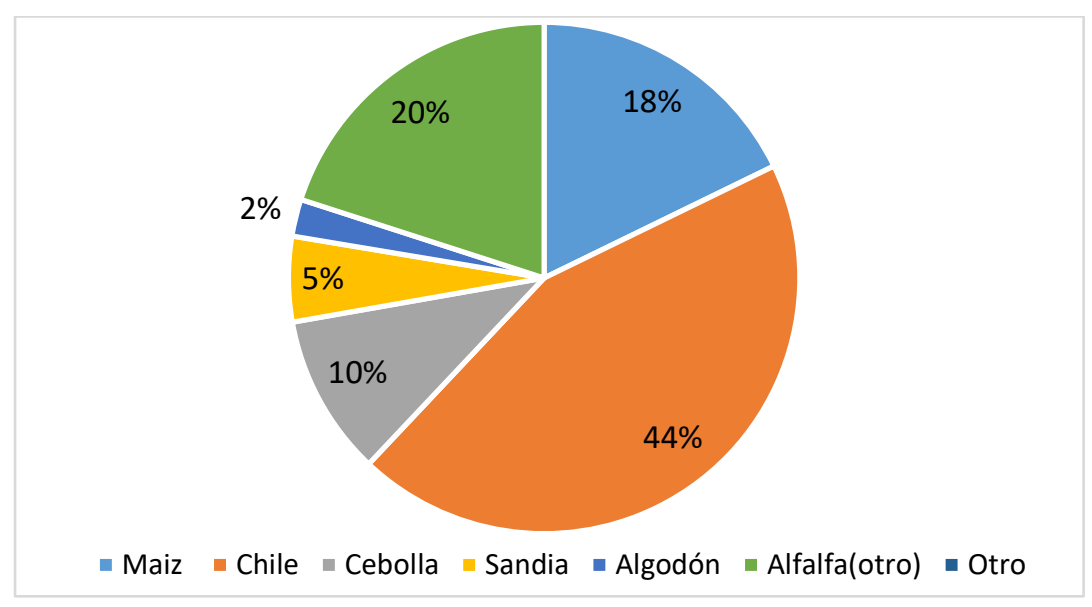

Figura 1. Porcentaje de producto sembrado, Elaboración propia.

En México existen más de 100 variedades de chile de los cuales los más comunes son el chile verde, el habanero, el pimiento morrón, el jalapeño y el chile poblano, los cuales se 
producen en más de 150 mil hectáreas divididas a lo largo de casi de toda la República, donde estados como Chihuahua, Zacatecas, San Luis Potosí y Michoacán son los principales productores con un total de más de dos millones de toneladas de chile al año, equivalentes al 77 por ciento del volumen total a nivel nacional (El chile poblano, popular en la cocina mexicana, 2018).

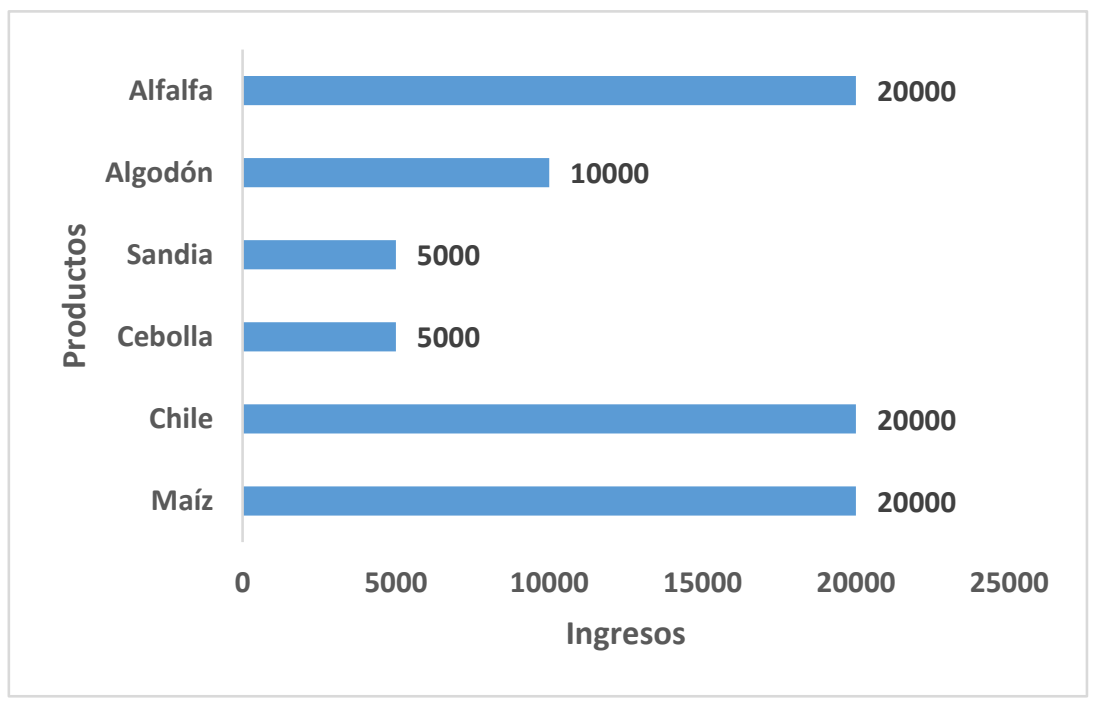

Figura 2. Productos que generan más ingresos, Elaboración propia.

La mayoría de los municipios del Estado de Chihuahua dependen de la agricultura y de las actividades conexas para una parte significativa de sus medios de vida. Dentro del Municipio de Julimes, se obtuvo que el maíz, chile y alfalfa, son los productos que generan más ingresos, se encuentran en el rango de 20 mil a más. Los productos subsecuentes que generan ingresos son el algodón con 10 mil, y la sandía y cebolla con 5 mil (Figura 3).

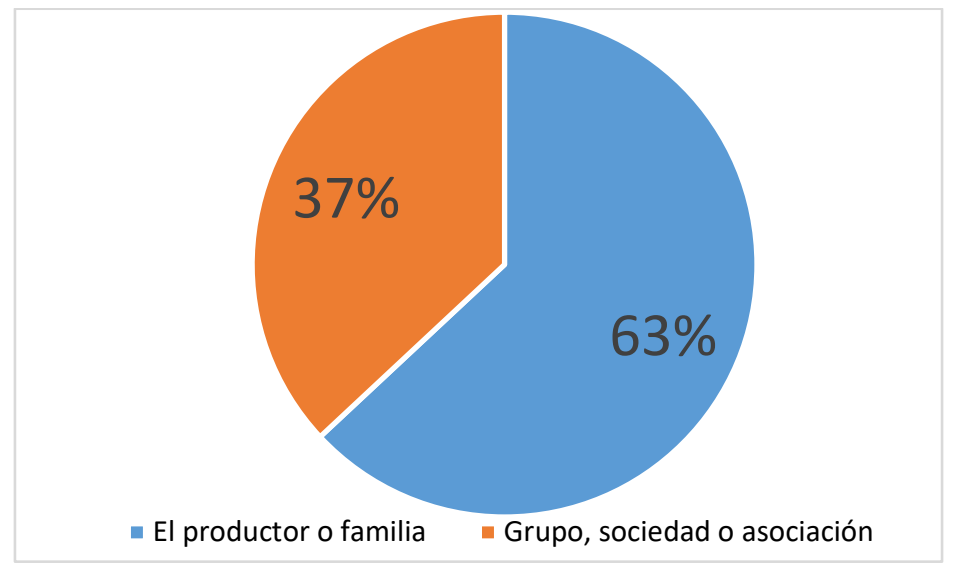

Figura 3. Personas que manejan los terrenos agrícolas, Elaboración propia. 
Los terrenos agrícolas familiares son sistemas de producción de alimentos para el autoconsumo que contribuyen a mejorar la seguridad alimentaria y la economía de los pequeños agricultores. De acuerdo a los datos arrojados por la figura 4 , el $63 \%$ de los terrenos son manejados por la familia, y el $37 \%$ por un grupo, sociedad, o asociación. Lo cuál implica que la responsabilidad para el manejo, la implementación y el control de las tierras, se comparte por los miembros de la familia.

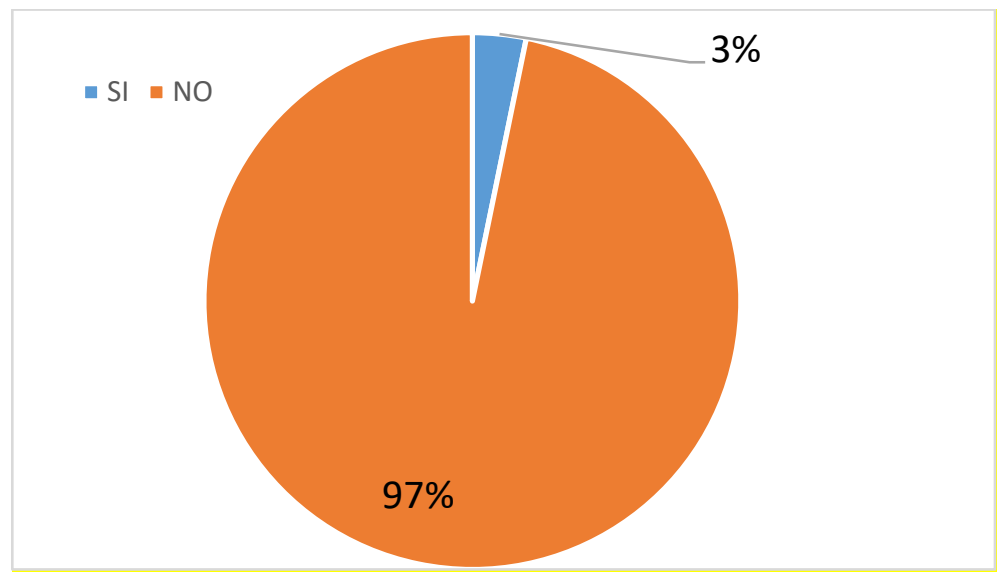

Figura 4. Pertenece a una organización, Elaboración propia.

Julimes, es un municipio rural, el cual su principal actividad económica es la agricultura, el $97 \%$ de los productores que representan esta actividad, mencionaron no pertenecer a ninguna organización, mientras que el 3\% menciono que sí (Figura 5). Pertenecer a una organización es importante ya que, mediante la aportación de cada productor dentro de ésta, permitirán elaborar una metodología de trabajo, para lograr un fin específico, el cuál es buscar la mejor calidad de vida para los productores del municipio de Julimes.

En el Estado de Santa Catarina los agricultores participan en varias instituciones sociales y están organizados de distintas maneras. Las
Comisiones de las Microcuencas. Cada microcuenca forma una Comisión con el objetivo de manejarse autónomamente; está constituida solo por agricultores que representan a las distintas comunidades. Otros objetivos de la Comisión son el de evaluar si los incentivos de los proyectos han sido correctamente aplicados, participar en la elaboración y supervisión de los trabajos de mejoramiento de caminos, coordinar acciones tales como la compra de maquinarias, equipos e insumos para uso comunal y administrar las actividades comunales tales como la operación de los secadores de granos (De Freitas \& Organització de les Nacions Unides per a l'Agricultura i l'Alimentació, 2000). 


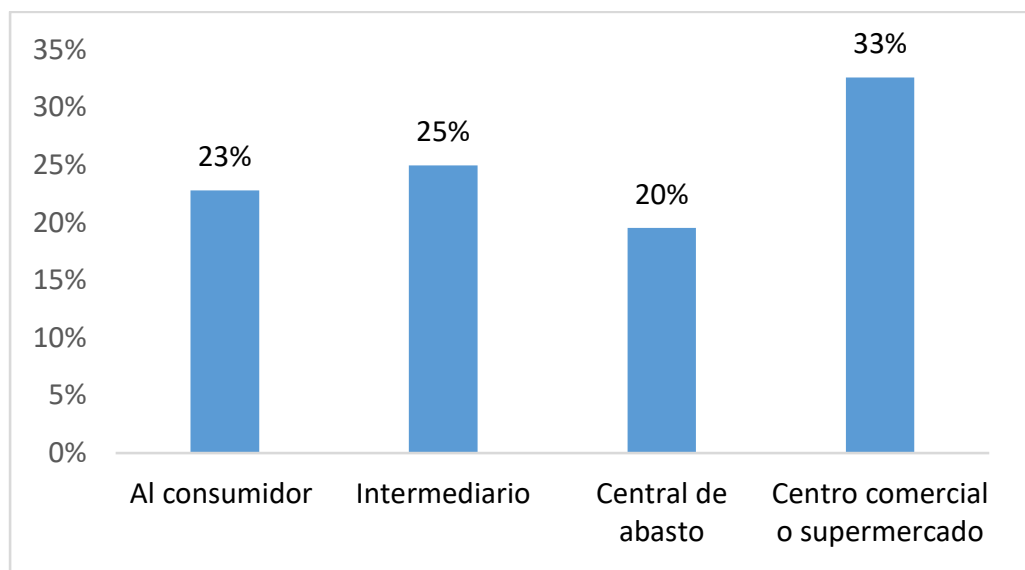

Figura 5. De los cultivos, la producción la vendió, o la espera vender, Elaboración propia.

La comercialización de la mayoría de los productos agropecuarios sigue enfrentada a una larga cadena de intermediarios que, en opinión de los productores, perjudica tanto al agricultor como al consumidor final. A pesar de que las grandes cadenas de supermercados y los expendios especializados han comenzado a entenderse directamente con los agricultores para el suministro de algunos productos, este sistema cubre apenas a unos pocos alimentos. De acuerdo a la encuesta realizada, se obtuvo un $33 \%$ que los agricultores esperan vender sus productos a un supermercado o un centro comercial, el 25\% a un intermediario, el 23\% al consumidor y el $20 \%$ a una central de abastos (Figura 6), por lo cual, la gente busca un mayor ingreso económico, para así tener una mejor calidad de vida.

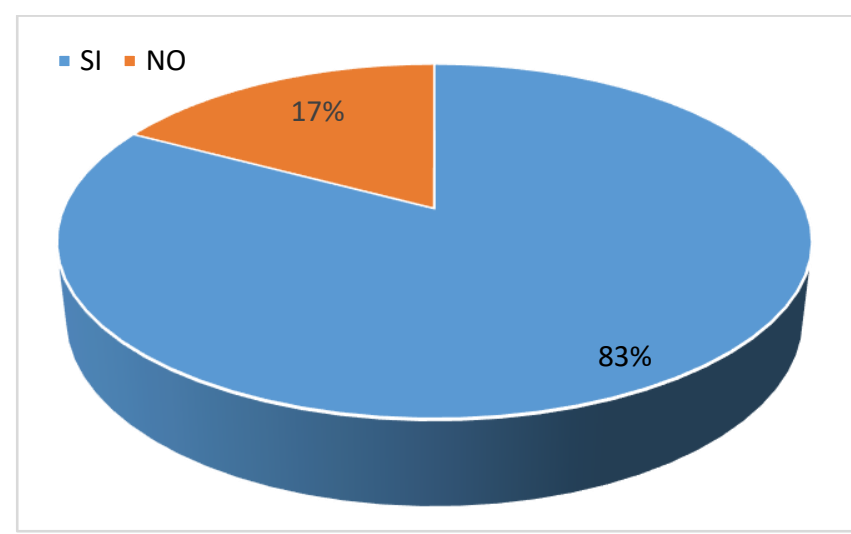

Figura 6. Le gustaría pertenecer a la cooperativa agrícola, Elaboración propia.

Las cooperativas permiten compartir los costos y beneficios de servicios a los que no podrían tener acceso por si solos (De Freitas \& Organització de les Nacions Unides per a l'Agricultura i l'Alimentació, 2000).

El $83 \%$ de los pequeños productores opinaron que les gustaría pertenecer a una cooperativa agrícola, mientras que tan solo el $17 \%$ opinó que no les gustaría (Figura 7). El tema de una cooperativa es de suma importancia, ya que a través de estas se puede obtener tecnología, información, capacitación, crédito, e incluso una mejor calidad de vida, obteniendo el mayor de los ingresos mediante la venta de sus productos cosechados. 
Jaime-Talamantes et al., 2012

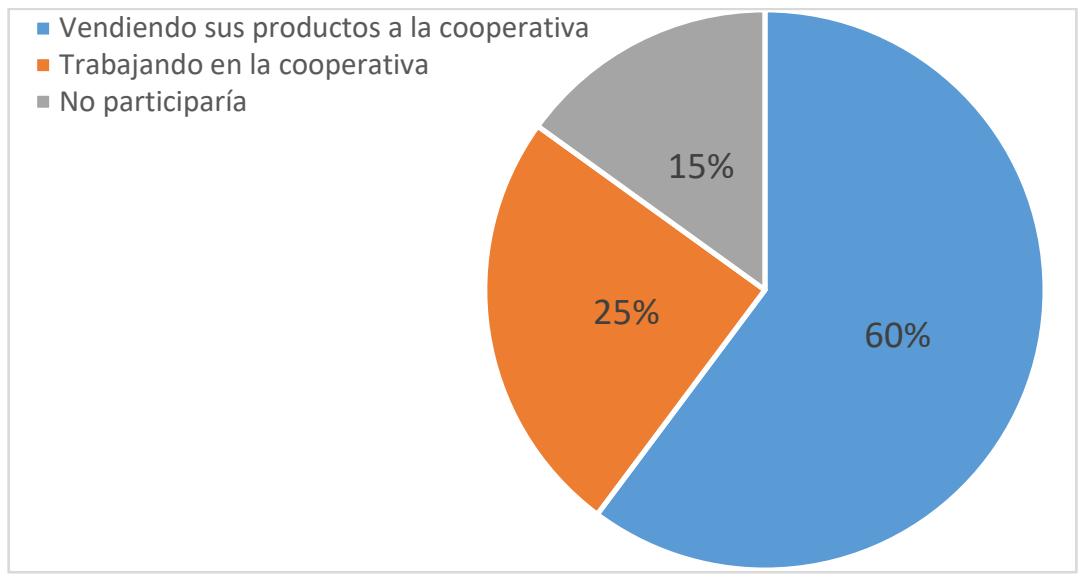

Figura 7. Participación en la Cooperativa Agrícola, Elaboración propia.

Como ya fue mencionado en la figura 8 , una cooperativa permite que pequeños, medianos y grandes productores se asocien, acabando con ese modelo en el que pareciera que los pequeños productores solo puedan quedarse en la fase de producción, mientras los beneficios de transformar, comercializar y quedarse con el valor agregado solo pueden hacer los grandes empresarios. De acuerdo a los porcentajes obtenidos en la figura anterior, el cuál el $83 \%$ de la población participaría dentro de ésta. El $60 \%$ de los campesinos vendería sus productos a la cooperativa, el $25 \%$ trabajaría dentro de esta, mientras que el $15 \%$ respondió que no participaría. De acuerdo a los resultados de la encuesta, este $15 \%$ se debe a que los grandes empresarios, tienden a tener la oportunidad de tener gente trabajando sus terrenos, bajo un sueldo, y los productos cosechados, venderlos a grandes empresas, como la Central de Abastos, La Costeña, etc. Un caso de éxito de cooperativas en el sector agrícola es Colanta, que inició con la asociación de 60 campesinos y hoy tiene 7.000 trabajadores asociados y 12.000 productores.

Para mantenerse en el tiempo, la compañía ha mantenido la innovación en sus procesos y productos. (Munevar Jaime, 2016).

\section{CONCLUSIONES}

La comercialización de la mayoría de los productos agropecuarios sigue enfrentada a una larga cadena de intermediarios que, en opinión de los productores, perjudica tanto al agricultor como al consumidor final. Las cooperativas han surgido como una forma de organización diferente a las formas tradicionales existentes en el mundo, con una finalidad de bien común, y prescindiendo del ánimo de lucro que caracteriza a otras formas organizativas con las que compite en el mercado. La presente investigación desarrolla un análisis que muestra el potencial de implantar una cooperativa agrícola, generando un trabajo digno y sostenible en un ambiente de bienestar, vinculando a los actores localmente activos con el aprovechamiento de sus recursos endógenos, que les permita el logro de los objetivos económicos y sociales. Así mismo, y de acuerdo a la información recabada en nuestras encuestas de campo, observamos que, la importancia que la agricultura tiene, se hace evidente no solo al proporcionar alimentos y materias primas, si no también oportunidades de empleo a una importante cantidad de población.

La agricultura engloba a un $80 \%$ de los terrenos agrícolas en el Municipio de Julimes, esto equivale a 13,066 ha en promedio. El producto 
Jaime-Talamantes et al., 2012

con más siembra por año es el chile con un 44\%, alfalfa $20 \%$ y el maíz $18 \%$. Sin embargo, estos son los productos que generan más ingresos, se encuentran en el rango de 20 mil pesos o más.

Dentro de la población, existen tres personas con los mayores ingresos del municipio, estas personas, tienen su empresa, con sus trabajadores, por los cuales, sus productos son vendidos a supermercados o mercados pequeños de los diferentes municipios aledaños.

Por otro lado, el origen de este análisis, nace, en el momento que se encuentra la situación de una persona, destinada a comprar las pequeñas cantidades de diferentes productores, a un bajo precio, y estos productos poder ser comercializados en una central de abastos supermercados o empresas. A esta persona le llaman coyote. Por último, y basados en lo expuesto, podemos concluir que las oportunidades que brinda la cooperativa agrícola como forma alternativa de organización son múltiples, teniendo en cuenta que este tipo de asociación, permitirá integrar diferentes actividades en un amplio espectro operativo, con la finalidad de brindar al productor la posibilidad de comercializar su producción, obteniendo el ingreso deseado. Asimismo, el principio de integración cooperativa y la solidaridad permiten superar restricciones que surgen del modelo económico y social que impera en estos tiempos.

\section{LITERATURA CITADA}

De Freitas, V. H., \& Organització de les Nacions Unides per a l'Agricultura il'Alimentació. (2000). Manejo del suelo en pequeñas fincas: Estrategias y métodos de introducción, tecnologías y equipos: experiencias en el estado de Santa Catarina, Brasil.

El chile poblano, popular en la cocina mexicana. (2018). https://www.gob.mx/ agricultura/articulos/el-chile-poblano-reyde-los-rellenos

Favi, F. P., \& Martínez, c. R. (2013).

Cooperativismo como herramienta del desarrollo territorial rural: experiencias concretas, desafíos y aprendizajes. 1(2), 15.INEGI. (2005). Prontuario de Información Geográfica Municipal. 9.

Munevar Jaime. (2016). La importancia de las cooperativas agropecuarias en el desarrollo del campo. https://www.larepublica.co/especiales/es pecial-cooperativismo/la-importanciade-las-cooperativas-agropecuarias-enel-desarrollo-del-campo-2753702 
Copyright (c) 2020 Mónica Arlette Jaime Talamantes, Arwell Nathán Leyva Chávez, Anabel Ortega Rodríguez, Amanda García Muñoz y Francisco Javier Piña Ramírez

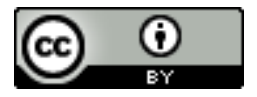

Este texto está protegido por una licencia Creative Commons $\underline{4.0 .}$

Usted es libre para Compartir — copiar y redistribuir el material en cualquier medio o formato-y Adaptar el documento —remezclar, transformar y crear a partir del material- para cualquier propósito, , incluso para fines comerciales, siempre que cumpla la condición de:

Atribución: Usted debe dar crédito a la obra original de manera adecuada, proporcionar un enlace a la licencia, e indicar si se han realizado cambios. Puede hacerlo en cualquier forma razonable, pero no de forma tal que sugiera que tiene el apoyo del licenciante o lo recibe por el uso que hace de la obra.

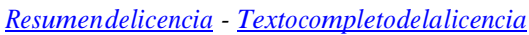

